\title{
Construction and Test of the Top and Bottom Counting Detectors for the ISS-CREAM Experiment
}

\author{
J.M. Park* (for the ISS-CREAM Collaboration') \\ Dept. of Physics, Kyungpook National University, Daegu 702-701, Republic of Korea \\ E-mail: jeongmin1024@gmail.com
}

\begin{abstract}
It is important to measure the cosmic ray spectrum to understand the origin, acceleration and propagation mechanisms of high-energy cosmic rays. The Cosmic Ray Energetics And Mass experiment will be launched in 2016 on the International Space Station (ISS-CREAM) to measure cosmic ray elemental spectra up to energies beyond the reach of balloon instruments. The main goal of the Top Counting Detector (TCD) and Bottom Counting Detector (BCD) is to separate electrons from protons using the difference between electromagnetic and hadronic shower shapes in the energy range of $300 \mathrm{GeV} \sim 800 \mathrm{GeV}$. The T/BCD consist of a plastic scintillator attached to $20 \times 20$ photodiodes. The active detection areas in the T/BCD are $500 \times 500 \mathrm{~mm}^{2}$ and 600 $\times 600 \mathrm{~mm}^{2}$, respectively. The TCD is located between the ISS-CREAM carbon target and its calorimeter, and the $\mathrm{BCD}$ is located below the calorimeter. Before integration with the payload, the T/BCD were assembled and conducted vibration and thermal vacuum tests to confirm the safety of the T/BCD under a space environment. The noise and gain of the T/BCD were tested with flight electronics, and the capability of the T/BCD to separate electrons from protons has been studied with a GEANT3 simulation. We present the design, construction, performance and simulation results of the T/BCD.
\end{abstract}

The 34th International Cosmic Ray Conference,

30 July- 6 August, 2015

The Hague, The Netherlands

\footnotetext{
*Speaker.

${ }^{\dagger}$ http://cosmicray.umd.edu/iss-cream/collaboration
} 


\section{Introduction}

The cosmic ray electron spectrum shows a interesting structure at around $600 \mathrm{GeV}[1,2,3]$. In making direct space-based observation, the separation of electrons from protons is very important since the proton flux is over 100 times higher than the electron flux in the region [4].

ISS-CREAM experiment [5] will be launched in 2016. ISS-CREAM plans to measure the cosmic ray elemental energy spectra from $10^{12} \mathrm{eV}$ to $>10^{15} \mathrm{eV}$ [6]. ISS-CREAM consists of a Silicon Charge Detector (SCD), a carbon target, a tungsten/scintillator sampling calorimeter (CAL), the TCD/BCD [5] and a Boronated Scintillator Detector (BSD).

The T/BCD are designed for separating the electrons from the protons by using different shower shapes in electromagnetic and hadronic interactions in the detectors in the energy range of $300 \mathrm{GeV} \sim 800 \mathrm{GeV}$ [7]. The T/BCD also provide a redundant ISS-CREAM instrument trigger beyond that from the CAL, and a low-energy electron trigger [6]. In this paper the design, construction, performance and simulation results of the T/BCD are described.

\section{Design and Construction}

For assurance the detector safety and space qualification for launch and ISS deployment and operations, detailed environmental tests and simulations are required. For instance, the mechanical safety and integrity of the detector enclosures are tested at various vibration frequencies, acceleration and temperature conditions, and are also simulated by using the Solidworks program [8].
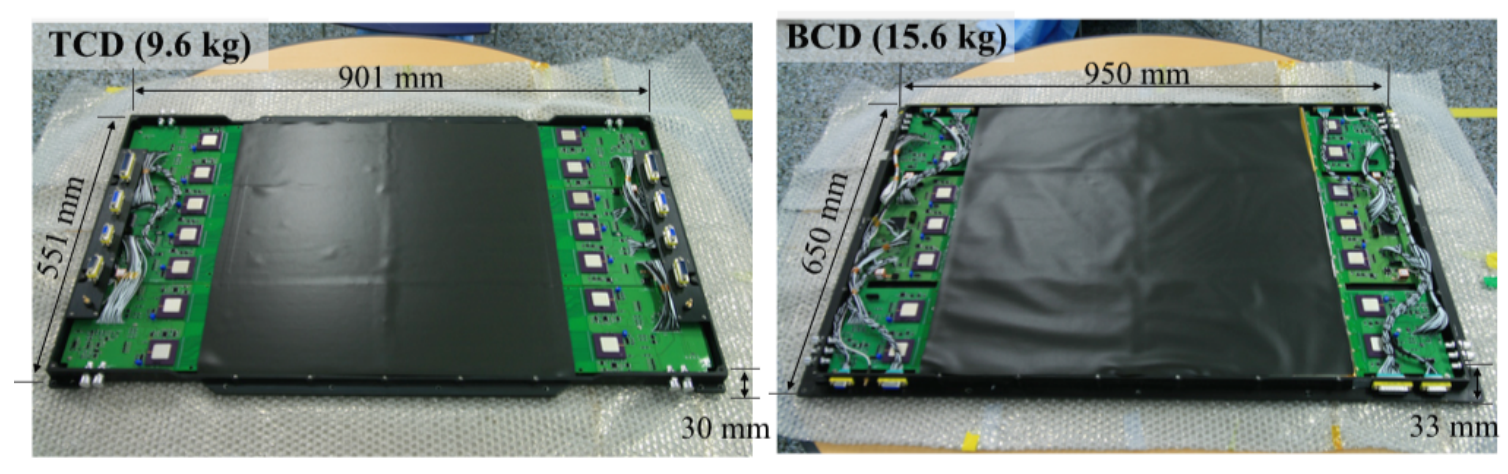

Figure 1: Assembled TCD (left) and BCD (right), before insertion into their mechanical enclosures.

The dimensions of the TCD are $901 \times 551 \times 30 \mathrm{~mm}^{3}$ and those of the BCD are $950 \times 650$ $\times 33 \mathrm{~mm}^{3}$. The T/BCD each consisted of a EJ-200 plastic scintillator (Eljen Technology) and a $20 \times 20$ silicon photodiode (PD) array, respectively. The plastic scintillator was attached to the PDs using DC93-500 optical silicone adhesive (Dow Corning), and the PDs were attached to printed circuit boards by using an Eccobond $56 \mathrm{C}$ conductive epoxy (Hysol). To prevent any light loss from the plastic scintillator, we wrapped in an enhanced specular reflector light reflector (3M Vikuiti). We used poron foam (Rogres corporation) between the aluminum enclosure and detectors for cushion the detector elements during launch conditions. The final detector assemblies without covers are shown in Fig. 1. The masses of the T/BCD are $9.6 \mathrm{~kg}$ and $15.6 \mathrm{~kg}$, respectively [9]. 
The T/BCD electronics system comprised two mother boards and four daughter boards. Each mother board had three VA-TA chips [10], one ACTEL chip, and two DC-DC converters. Each daughter board had two VA-TA chips to read the PD signals [11]. Figure 2 shows a schematic layout of the electronics systems.

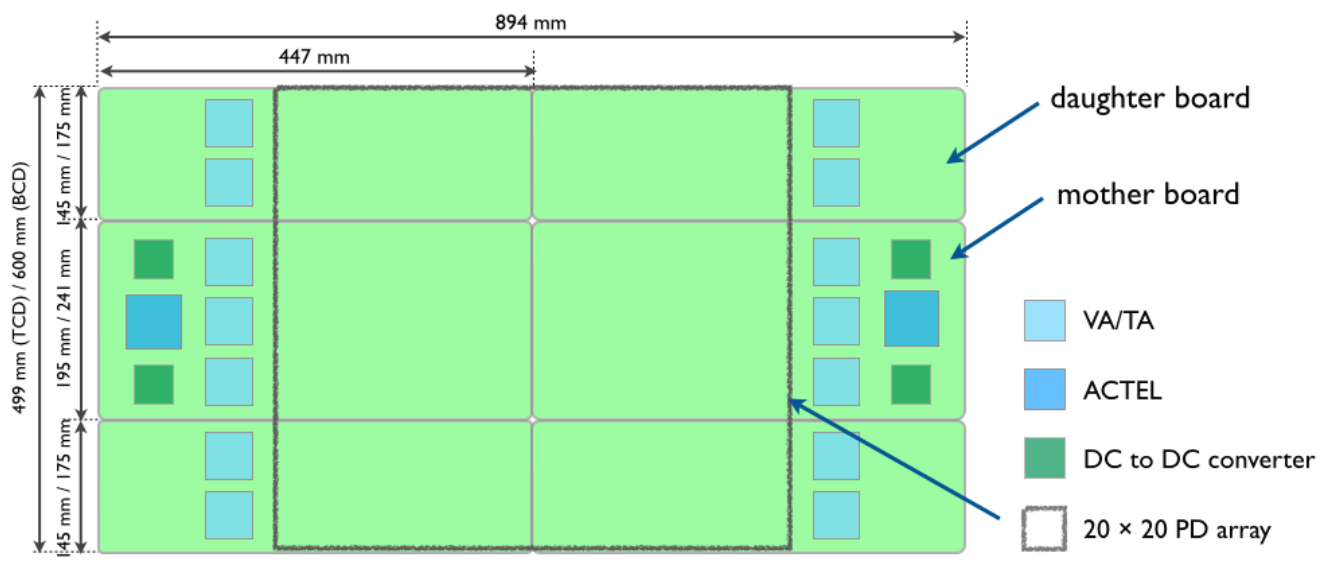

Figure 2: Layout of the T/BCD electronics systems.

\section{Environmental Tests}

The vibration test of the T/BCD was performed at Keimyung University in Korea [12]. Figure 3(a) shows the TCD on the shaker. The goal of this test was to verify the safety of the detector under
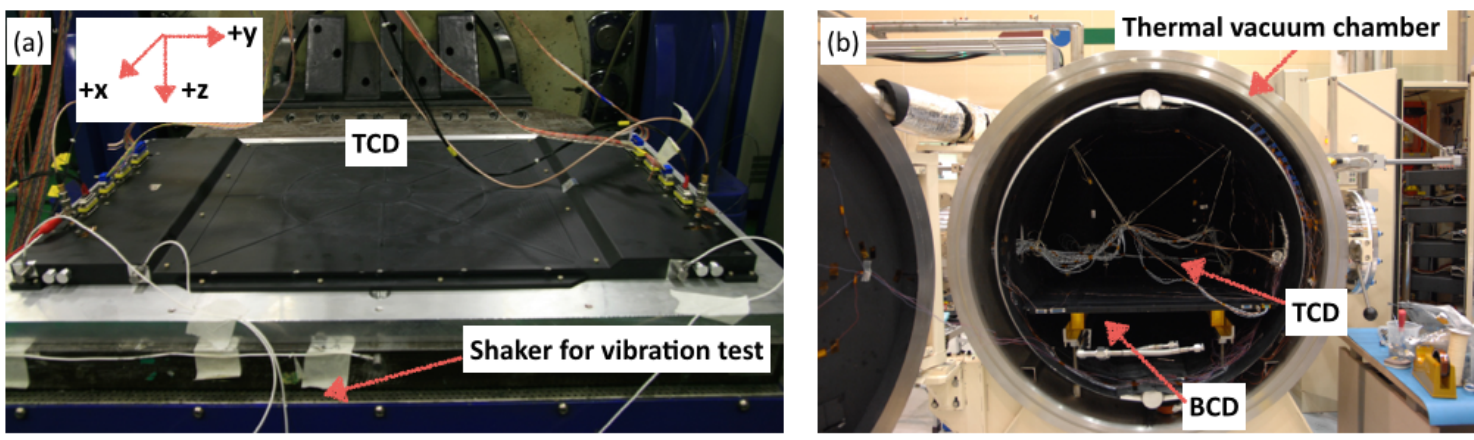

Figure 3: Experimental setup for the (a) vibration test and (b) thermal vacuum test.

maximum expected launch conditions. Signature sine sweep, swept sine, sine burst, or random vibration conditions were applied in turn. During the vibration tests, we compared the sine sweep results, which showed resonance frequencies of the T/BCD, to the functional test results to verify the mechanical integrity of the T/BCD. Following the random vibration test at the maximum level of $+3 \mathrm{~dB}$, which checked the structural response of the detectors, the results of the sine sweep and function tests were similar to the pre-test ones, as shown in Fig. 4. This qualified the detectors to survive at the launch conditions [11].

The thermal vacuum test of the T/BCD was performed at the Korea Aerospace Research Institute (KARI) [13]. Figure 3(b) shows the experimental setup for the thermal vacuum test. All 
(a)

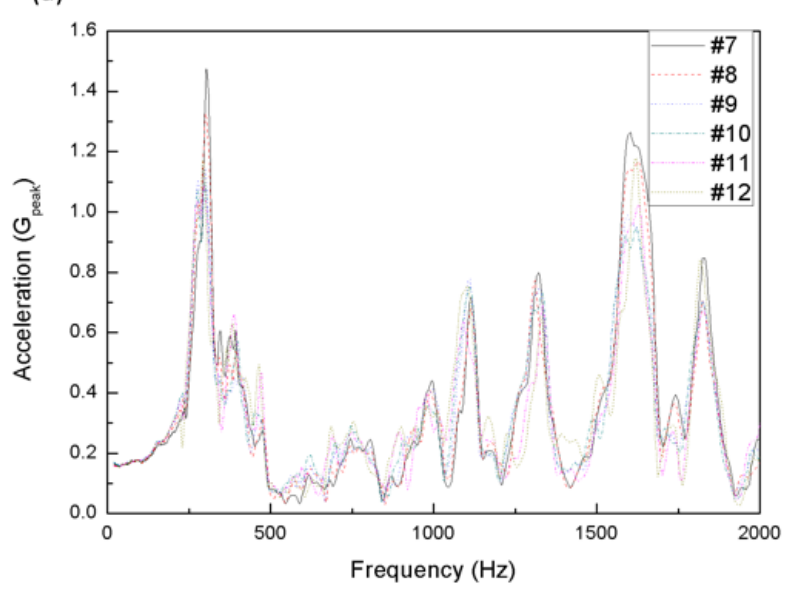

(b)

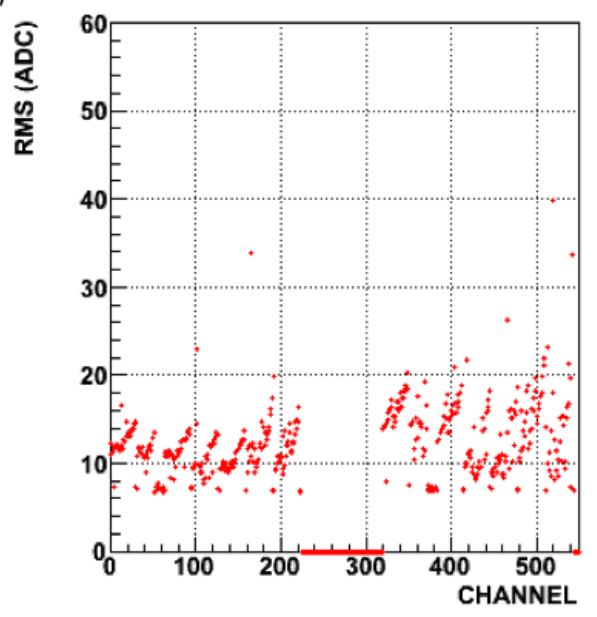

Figure 4: (a) Acceleration distributions of the BCD along the z-axis as a function of frequency during the sine sweep tests, which were performed between random vibration tests at different test levels. (b) Functional test results of the TCD after the vibration test along the z-axis.

detectors need to maintain components within the survival temperature range during the tests, i.e., between $-40{ }^{\circ} \mathrm{C}$ and $+55^{\circ} \mathrm{C}$ (the normal operating range being $-20{ }^{\circ} \mathrm{C}$ to $+40{ }^{\circ} \mathrm{C}$ at $1 \times 10^{-5}$ Torr). The T/BCD operated normally during the thermal vacuum test, but had problems such as power drops at a low temperature. This problem was caused by the fact that the original current circuits was designed for two VA/TA chips, but the motherboard has three of them. Thus the available current was insufficient, which was remedied by changing the current limiter for the three ASIC chips $[9,11]$.

\section{Integration and Performance Tests at the Wallops Flight Facility}

After verifying the detector's safety under launch conditions, the T/BCD were integrated into the ISS-CREAM payload at the NASA Wallops Flight Facility (WFF). Figure 5 shows photographs of the ISS-CREAM instrument before the SCD integration.

The noise, gain and muon response of the T/BCD were measured using the flight electronics to test the response to a minimum-ionizing particle (MIP). Figure 6 shows the noise (left) and gain levels (right) of the T/BCD. The pedestals' root mean square (RMS) value of the T/BCD was measured to be $<15$ ADC channels on average. The gain of each channel is about 280 DAC/ADC.

The muon response of the T/BCD was measured by using an external muon trigger system. Figure 7(a) shows the test results in which data were collected after specification cuts. The signal to noise ratio (SNR) is defined as

$$
S N R=M I P_{\text {signal }} / \sigma_{\text {pedestal }}
$$

The $\mathrm{MIP}_{\text {signal }}$ corresponds to the energy when the MIP penetrates the detector and $\sigma_{\text {pedestal }}$, $15 \mathrm{ADC}$ channels $(\simeq 0.042 \mathrm{MeV})$, is the width of the pedestal distribution. When the MIP penetrates a $1 \mathrm{~cm}$ thick plastic scintillator and a $650 \mu \mathrm{m}$ thick silicon sensor vertically, the energy 
(a)

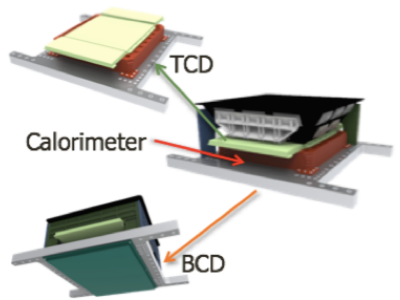

(c)

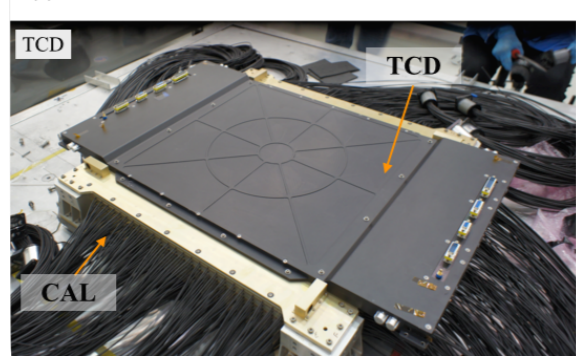

(b)

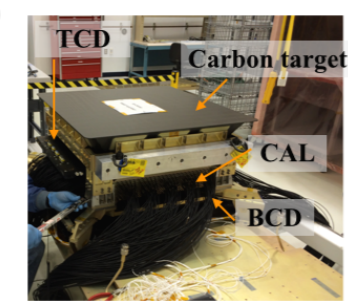

(d)

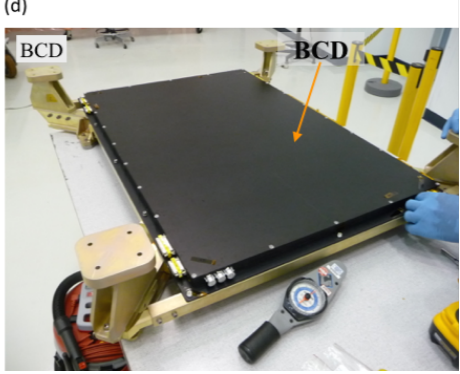

Figure 5: (a) Illustration of the T/BCD, and photographs of the (b) ISS-CREAM instrument without the SCD at the WFF, (c) the TCD and (d) the BCD.
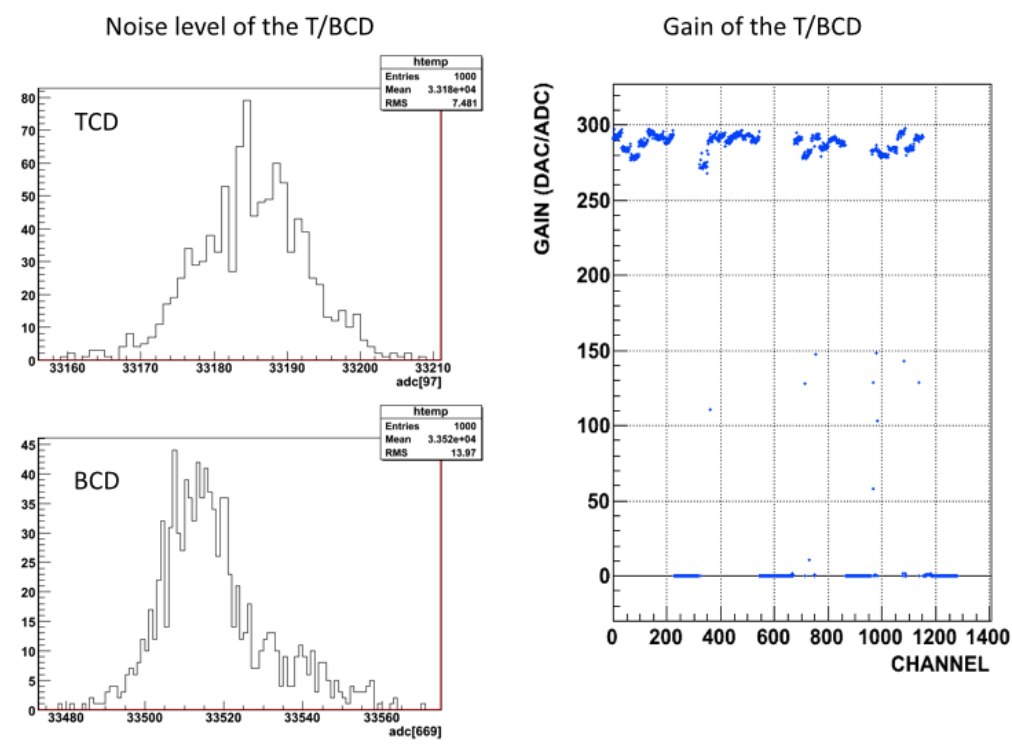

Figure 6: The noise (left) and gain levels (right) of the T/BCD.

deposited about $9.6 \mathrm{fC}$. Since we used an external trigger system, the data included muon events which had random incident directions so that the deposited average energy is about $13.4 \mathrm{fC}(\simeq$

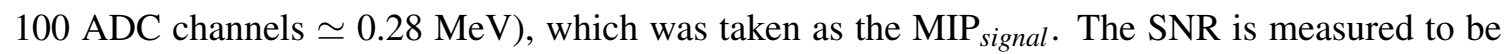
6.7. Figure 7(b) shows a muon response distribution from a Monte Carlo simulation with a noise smearing. During the measurement of muon events, the noise level and MIP energy were similar to the simulation result. According to these tests, the T/BCD are found to be sensitive to single MIP. 
(a)
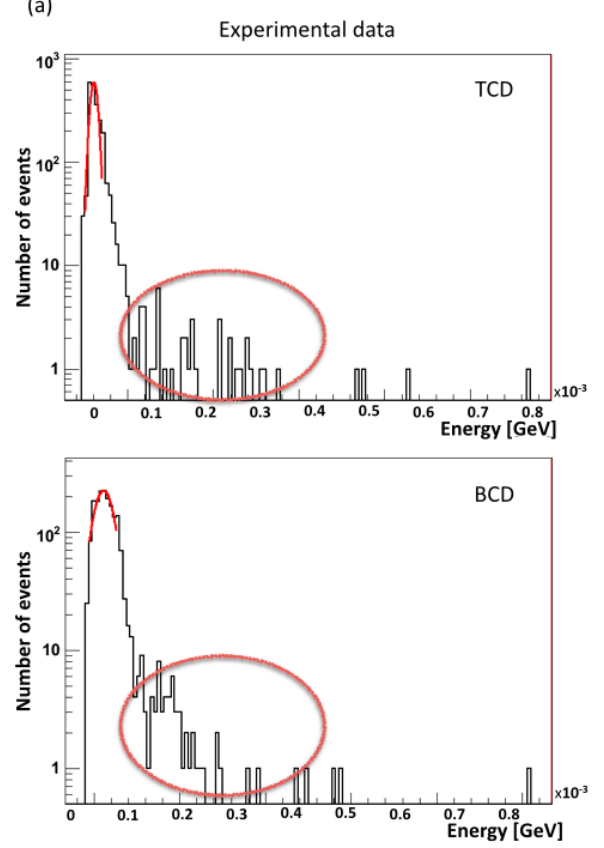

(b)

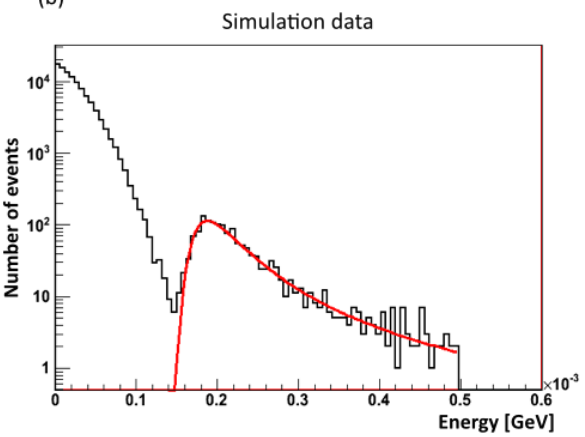

Figure 7: Cosmic rays muon responses in (a) experimental data and (b) simulated events for the T/BCD.

\section{5. e $(\gamma) / p$ Separation Study Using a GEANT3 Simulation}

The main goal of the T/BCD is to separate electrons from protons by using the difference in shower shapes of the electromagnetic and hadronic interactions. An electromagnetic shower shape is shorter and narrower than that of a hadronic shower [14]. We generated $300 \mathrm{GeV}$ electrons and $900 \mathrm{GeV}$ protons at vertical incidence by using a GEANT3 simulation [15]. We considered the electronics noise level for the VA and trigger by using previous CREAM experiment results [16]. In the simulation, we used different cuts for the TCD and BCD. Most electrons lose their energies in the TCD and the hit distributions are very narrow. By comparing the number of hits and distributions between electrons and protons, we can separate electrons from protons. The hit distributions of $300 \mathrm{GeV}$ electrons and $900 \mathrm{GeV}$ protons in the T/BCD are shown in Fig. 8(a). The RMS distribution in the TCD and $\mathrm{F}_{\text {factor }}$ distribution in the BCD are shown in Fig. 8(b). The RMS is defined as

$$
R M S^{2}=E_{T C D_{i}} \times\left\{\left(x_{i}-x_{c}\right)^{2}+\left(y_{i}-y_{c}\right)^{2}\right\}
$$

where $\mathrm{x}_{i}, \mathrm{y}_{i}$ are the coordinates of the center of the energy deposited in the sensor, $\mathrm{x}_{c}, \mathrm{y}_{c}$ are the coordinates of the energy center in the TCD, and $\mathrm{E}_{T C D_{i}}$ is the energy deposited in the $i$ th sensor of the TCD. The $\mathrm{F}_{\text {factor }}$ is defined as

$$
F_{\text {factor }}=R M S^{2} \times E_{B C D} / E_{C A L},
$$

where $\mathrm{E}_{B C D}$ is the energy deposition in the $\mathrm{BCD}$ and $\mathrm{E}_{C A L}$ is the total energy deposition in the CAL. These cuts are similar to those of the ATIC experiment analysis methods [3].

The proton rejection factor is defined as 
(a)
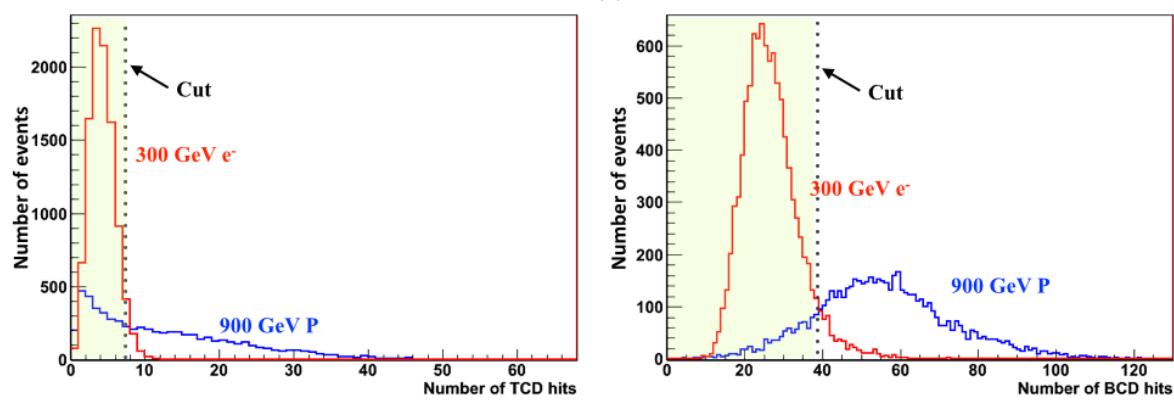

(b)
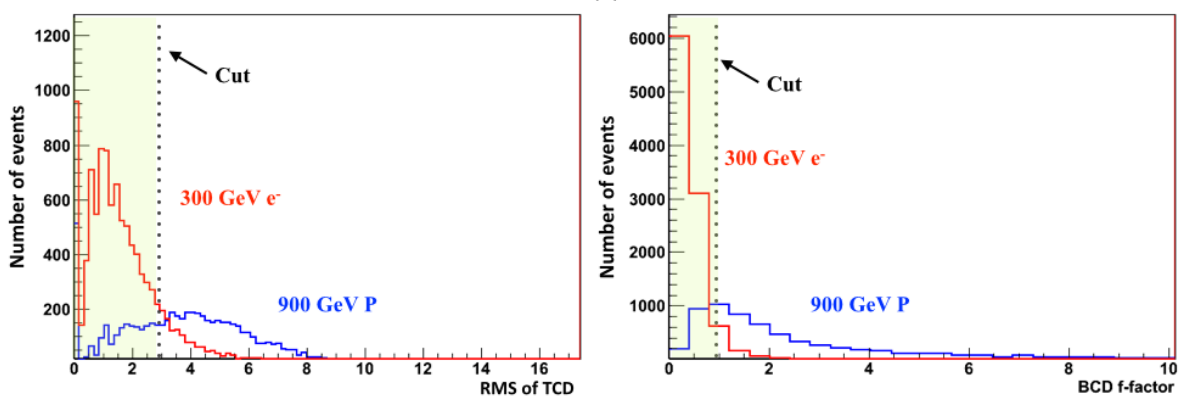

Figure 8: Electron/proton separation by using (a) the number of hits and (b) the RMS and $\mathrm{F}_{\text {factor }}$ in the GEANT3 simulation.

Proton rejection factor $=$ Num. of incident proton $/$ Num. of selected proton.

The electron efficiency and the proton rejection factor are found to be $73.6 \%$ and 100 , respectively, by applying these cuts. We employed the log-likelihood fit shown in Fig. 9 for four cut parameters to improve the proton rejection power. Figure 9 shows the $300 \mathrm{GeV}$ electron and $900 \mathrm{GeV}$ proton distributions using the likelihood fit. The electron efficiency is $62.5 \%$ and the proton rejection factor is doubled to 222 .

\section{Conclusion}

The T/BCD are developed for the ISS-CREAM experiment and the main goal of the T/BCD is the separation of the signals from protons by using shower shape differences. The T/BCD each consist of 400 photodiodes and a plastic scintillator, respectively. The vibration and thermal vacuum tests were performed to assure the safety of the T/BCD under a space environment. The test results showed that the T/BCD are flight qualified. The T/BCD successfully integrated into the ISS-CREAM payload at the WFF and their MIP detection capability was tested by measuring the noise, gain, and muon response of the detectors. The SNR was measured to be 6.7 for muon signals and the possibility of single MIP detection is addressed by comparing data with a Monte Carlo simulations. From our Monte Carlo study using four parameters (number of hits in the T/BCD, RMS in the TCD, $\mathrm{F}_{\text {factor }}$ in the $\mathrm{BCD}$ ) for $300 \mathrm{GeV}$ electron and $900 \mathrm{GeV}$ proton events, the electron efficiency and the proton rejection factor are found to be $62.5 \%$ and 222 , respectively. 


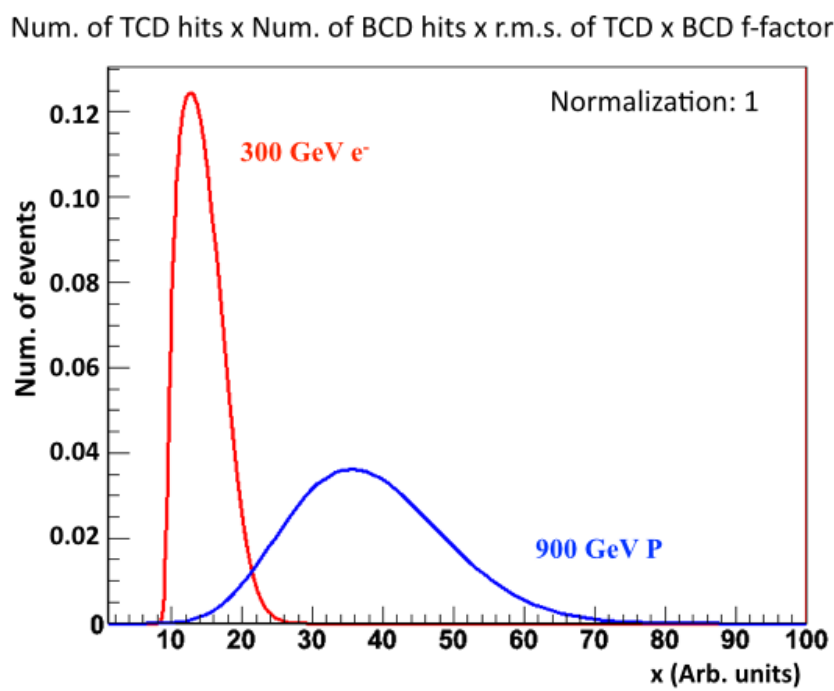

Figure 9: The $300 \mathrm{GeV}$ electron and $900 \mathrm{GeV}$ proton distributions using the likelihood fit.

\section{Acknowledgments}

The authors thank the NASA GSFC WFF for project management and engineering support, and the NASA JSC ISS Program Office for the launch support and the ISS accommodation. This work was supported in the U.S. by NASA grants NNX11AC52G, NNX08AC15G, NNX08AC16G and their predecessor grants, as well as by directed RTOP funds to NASA GSFC WFF. It is supported in Korea by the Creative Research Initiatives of MEST/NRF and by National Research Foundation Grants NRF-2014R1A2A2A01002734, NRF-2014R1A1A2006456. It is supported in France by IN2P3/CNRS and CNES and in Mexico by DGAPA-UNAM and CONACYT. The authors also thank H.S. Choi, Korea Institute of Industrial Technology, for contributions to the SCD thermal vacuum tests, M. Geske, Penn State, for contributions to the BSD, and M. A. Coplan, University of Maryland, contributions to CAL electronics vacuum tests.

\section{References}

[1] A.A. Abdo et al., Measurement of the Cosmic Ray $e^{+}+e^{-}$Spectrum from $20 \mathrm{GeV}$ to $1 \mathrm{TeV}$ with the Fermi Large Area Telescope, Physical Review Letters 102 (2009) 181101.

[2] The Alpha Magnetic Spectrometer Experiment http://www.ams02.org/.

[3] J. Chang et al., Resolving electrons from protons in ATIC, Advances in Space Research 42 (2008) 431-436.

[4] C.D. Dermer, Diffuse Galactic Gamma Rays from Shock-Accelerated Cosmic Rays, Physical Review Letters 109 (2012) 091101.

[5] E.S. Seo et al., Cosmic Ray Energetics and Mass for the International Space Staion, Advances in Space Research 53/10 (2014) 1451-1455.

[6] H.J. Hyun et al., Development of Top/Bottom Counting Detectors for the CREAM Experiment on the ISS, Proc. of the 33rd Int. Cosmic Ray Conf., Rio de Janerio, Brazil (2013) ID 1017. 
[7] J.M. Park et al., Results of Tests and Simulations for the Top Counting Detector and Bottom Counting Detector of the ISS-CREAM Experiment, Proc. of the 33rd Cosmic Ray Conf., Rio de Janerio, Brazil (2013) ID 1015.

[8] SolidWorks, Introducing, Solidworks corporation, Concord, MA (2002).

[9] Y.S. Hwang et al., Construction and Testing of a Top Counting Detector and a Bottom Counting Detector for the Cosmic Ray Energetics And Mass Experiment on the International Space Station, J. of Instrum. 10 (2015) P07018.

[10] VA32HDR14.2 and TA32CG3, Gamma Medica-Ideas, Norway.

[11] H.J. Hyun el al., Performances of photodiode detectors for top and bottom counting detectors of ISS-CREAM experiments, Nucl. Inst. Meth, 787 (2015) 134-139.

[12] The Center for Automotive Mechatronics Parts, Deagu, South Korea http://camp.kmu.ac.kr/.

[13] Korea Aerospace Research Institute, Deajeon, South Korea http://www.kari.re.kr/.

[14] W. R. Leo., Techniques for nuclear and particle physics experiments: a how-to approach, Springer 1994.

[15] ROOT Data Analysis Framework http://root.cern.ch/

[16] M.H. Lee et al., The CREAM Calorimeter: Performance In Tests and Flights, AIP conference proceedings 867 (2006) 167. 